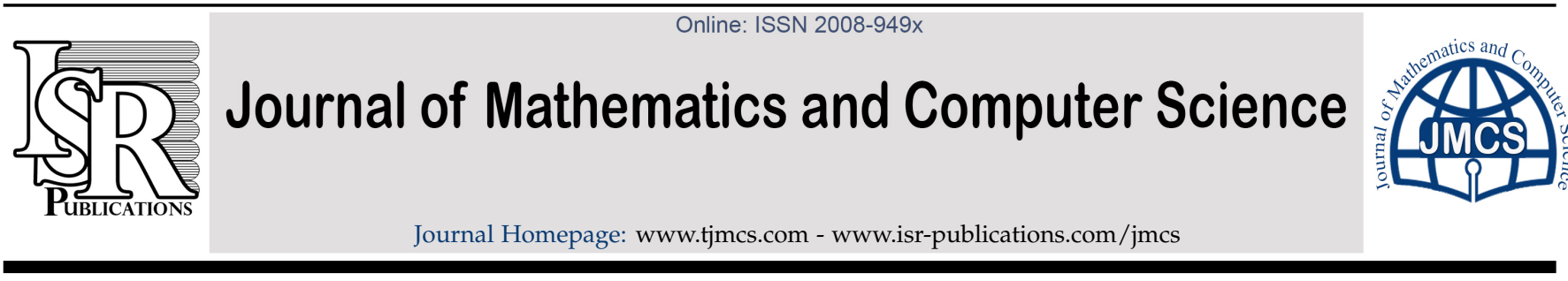

\title{
Jessen type functionals and exponential convexity
}

\author{
Rishi Naeem, Matloob Anwar* \\ School of Natural Sciences, National University of Sciences and Technology, Islamabad, Pakistan.
}

\begin{abstract}
In this paper, we introduce the extension of Jessen functional and investigate logarithmic and exponential convexity. We also give mean value theorems of Cauchy and Lagrange type. Several families of functions are also presented related to our main results. (C)2017 All rights reserved.
\end{abstract}

Keywords: Jessen functional, exponential convexity, mean value theorems. 2010 MSC: 26A24, 26A48, 26A51, 26D15.

\section{Introduction and preliminaries}

Let $E(\neq \emptyset)$ and $L$ be a linear class of real-valued functions $h: E \rightarrow \mathbb{R}$ having the properties:

$\mathrm{L}_{1}: h, k \in \mathrm{L} \Rightarrow(\alpha \mathrm{h}+\beta \mathrm{k}) \in \mathrm{L}$, for all $\alpha, \beta \in \mathbb{R} ;$

$\mathrm{L}_{2}: 1 \in \mathrm{L}$ that is if $\mathrm{h}(\mathrm{l})=1$ for $l \in E$, then $h \in \mathrm{L}$.

We also consider positive linear functionals $A: L \rightarrow \mathbb{R}$ possessing the properties:

$$
A_{1}: A(\alpha h+\beta k)=\alpha A(h)+\beta A(k) \text { for } h, k \in L, \alpha, \beta \in \mathbb{R} \text {; }
$$

$A_{2}: h \in L, h(l) \geqslant 0$ on $E \Rightarrow A(h) \geqslant 0$ ( $A$ is positive $)$.

The mapping $A$ is said to be normalized if

$$
A_{3}: A(1)=1 .
$$

By a weight function, we mean a mapping $\omega: E \times E \rightarrow \mathbb{R}^{+}$such that

$$
\begin{aligned}
& A(\omega(x, y))=1(\text { for each } y \text { in } E), \\
& B(\omega(x, y))=1(\text { for each } x \text { in } E),
\end{aligned}
$$

where $A$ and $B$ satisfy the properties $A_{1}, A_{2}$ and $A_{3}$.

\footnotetext{
*Corresponding author

Email addresses: rishi.naeem@sns.nust.edu.pk (Rishi Naeem), manwar@sns.nust.edu.pk (Matloob Anwar)
} doi:10.22436/jmcs.017.03.08 
Jessen in [12] (see also [20]) gave the generalization of Jensen's inequality for positive linear functionals: For a continuous convex function $\Psi: I \rightarrow \mathbb{R}$, where $I \subseteq \mathbb{R}$ and let $L$ satisfy properties $L_{1}, L_{2}$ on a nonempty set $E$. If $A$ is a positive linear functional on $L$ with $A(1)=1$, then for all $h \in L$ such that $\Psi(h) \in L$ we have $A(h) \in I$ and

$$
\Psi(A(h)) \leqslant A(\Psi(h)) .
$$

Jessen functional is the difference of above inequality, written as

$$
\Upsilon_{1}(\Psi)=A(\Psi(h))-\Psi(A(h)) .
$$

For other notable literature about Jensen's inequality and related results see $[1-3,8]$. The converse of Jessen's inequality [5] (see also [20]) is stated as:

Theorem 1.1. For a convex function $\Psi$ on an interval $\mathrm{I}=[\eta, \zeta](-\infty<\eta<\zeta<\infty)$ and let $\mathrm{L}$ satisfy properties $\mathrm{L}_{1}, \mathrm{~L}_{2}$ on a nonempty set $\mathrm{E}$. If $\mathrm{A}$ is an isotonic linear functional on $\mathrm{L}$ with $\mathrm{A}(1)=1$, then for all $\mathrm{h} \in \mathrm{L}$ such that $\Psi(\mathrm{h}) \in \mathrm{L}$ (so that $\eta \leqslant \mathrm{h}(\mathrm{l}) \leqslant \zeta$ for all $\mathrm{l} \in \mathrm{E}$ ), we have

$$
A(\Psi(h)) \leqslant \frac{\zeta-A(h)}{\zeta-\eta} \cdot \Psi(\eta)+\frac{A(h)-\eta}{\zeta-\eta} \cdot \Psi(\zeta) .
$$

In this paper we consider the functional defined from above inequality and is given as:

$$
\Upsilon_{2}(\Psi)=\frac{\zeta-A(h)}{\zeta-\eta} \cdot \Psi(\eta)+\frac{A(h)-\eta}{\zeta-\eta} \cdot \Psi(\zeta)-A(\Psi(h)) .
$$

The following theorem is the refinement of Jessen's inequality.

Theorem 1.2. Let $\mathrm{L}$ satisfy properties $\mathrm{L}_{1}$ and $\mathrm{L}_{2}$ on a nonempty set $\mathrm{E}$, and assume that $\Psi$ is a continuous convex function on an interval $\mathrm{I} \subseteq \mathbb{R}$. If $\mathrm{A}$ and $\mathrm{B}$ are positive linear functionals with $\mathrm{A}(1)=\mathrm{B}(1)=1$ and $\omega$ is a weight function (defined in (1.1)) then for all $h, h \cdot \omega \in L$ such that $\Psi(A(h \cdot \omega)), \Psi(h) \in L$ we have $A(h \cdot \omega), A(h) \in I$ and

$$
\Psi(A(h)) \leqslant B(\Psi(A(h \cdot \omega))) \leqslant A(\Psi(h)) .
$$

m-exponential convexity was firstly introduced by Pečarić and Perić in [19]. In [4, 7, 9, 10, 13-15, 18], the construction of m-exponentially convex functions is made through the method prescribed in [11]. The reader may refer to $[6,16,17,21]$ for the background of exponential convexity and mean value theorems.

In the next section, we prove the counterpart of the refined Jessen inequality and give its new version. In Section 3, we discuss m-exponential convexity of the functions associated with the linear functionals. We present different families of functions to investigate exponential convexity and log-convexity. Mean value theorems are also given in the last section.

Throughout paper, we assume $\omega$ to be a weight function which satisfies (1.1).

\section{Main results} $[\eta, \zeta]$.

Now we prove the counterpart of the inequality $\Psi(A(h)) \leqslant B(\Psi(A(h \cdot \omega)))$ for compact interval $I=$

Theorem 2.1. Let $\Psi$ be a convex function on $\mathrm{I}=[\eta, \zeta](-\infty<\eta<\zeta<\infty)$. Let $\mathrm{L}$ satisfy properties $\mathrm{L}_{1}, \mathrm{~L}_{2}$ on a nonempty set $\mathrm{E}, \omega$ is weight function and $\mathrm{A}, \mathrm{B}$ are isotonic linear normalized functionals on $\mathrm{L}$, then for all $h \cdot \omega \in \mathrm{L}$ such that $\Psi(A(h \cdot \omega)) \in L$ (so that $\eta \leqslant A(h \cdot \omega) \leqslant \zeta)$, we have

$$
B(\Psi(A(h \cdot \omega))) \leqslant \frac{\zeta-A(h \cdot \omega)}{\zeta-\eta} \Psi(\eta)+\frac{A(h \cdot \omega)-\eta}{\zeta-\eta} \Psi(\zeta) .
$$


Proof. From the definition of convex function

$$
\Psi(b) \leqslant \frac{c-b}{c-a} \Psi(a)+\frac{b-a}{c-a} \Psi(c), \quad(a \leqslant b \leqslant c, a<c) .
$$

Now set $a=\eta, b=A(h \cdot w), c=\zeta$ give

$$
\Psi(A(h \cdot \omega)) \leqslant \frac{\zeta-A(h \cdot \omega)}{\zeta-\eta} \Psi(\eta)+\frac{A(h \cdot \omega)-\eta}{\zeta-\eta} \Psi(\zeta) .
$$

Since B is isotonic linear and normalized functional, (2.1) holds.

The next theorems are our main results.

Theorem 2.2. Let $\Psi$ be a convex function on $\mathrm{I}=[\eta, \zeta](-\infty<\eta<\zeta<\infty)$. Let $\mathrm{L}$ satisfy properties $\mathrm{L}_{1}, \mathrm{~L}_{2}$ on a nonempty set $\mathrm{E}, \omega$ is weight function and $\mathrm{A}, \mathrm{B}$ are isotonic linear normalized functionals on $\mathrm{L}$, then for all $h, h \cdot \omega \in L$ such that $\Psi(A(h \cdot \omega)), \Psi(\eta+\zeta-A(h \cdot \omega)) \in L$ (so that $\eta \leqslant A(h \cdot \omega) \leqslant \zeta)$, we have

$$
\Psi(\eta+\zeta-A(h)) \leqslant \Psi(\eta)+\Psi(\zeta)-B(\Psi(A(h \cdot \omega))) .
$$

Proof. Since $\Psi$ is continuous and convex, the same is also true for the function $\Phi:[\eta, \zeta] \rightarrow \mathbb{R}$ defined by $\Phi(t)=\Psi(\eta+\zeta-t), t \in[\eta, \zeta]$. Then by the left hand side of the inequality of (1.2), we have

$$
\psi(A(h)) \leqslant B(\psi(A(h \cdot \omega))) .
$$

That is,

$$
\Psi(\eta+\zeta+A(h)) \leqslant B(\Psi(\eta+\zeta-A(h \cdot \omega))) .
$$

Applying Theorem 2.1, we obtain

$$
\begin{aligned}
B(\Psi(\eta+\zeta-A(h \cdot \omega))) & \leqslant \frac{\zeta-A(h \cdot \omega)}{\zeta-\eta} \Phi(\eta)+\frac{A(h \cdot \omega)-\eta}{\zeta-\eta} \Phi(\zeta) \\
& \leqslant \frac{\zeta-A(h \cdot \omega)}{\zeta-\eta} \Psi(\zeta)+\frac{A(h \cdot \omega)-\eta}{\zeta-\eta} \Psi(\eta) \\
& =\Psi(\eta)+\Psi(\zeta)-\left[\frac{\zeta-A(h \cdot \omega)}{\zeta-\eta} \Psi(\eta)+\frac{A(h \cdot \omega)-\eta}{\zeta-\eta} \Psi(\zeta)\right] \\
& \leqslant \Psi(\eta)+\Psi(\zeta)-B(\Psi(A(h \cdot \omega))) .
\end{aligned}
$$

The last statement follows from the fact that if $\Psi$ is concave, then $-\Psi$ is convex and $A, B$ are linear on L.

Theorem 2.3. Let $\Psi$ be a convex function on $\mathrm{I}=[\eta, \zeta](-\infty<\eta<\zeta<\infty)$. Let $\mathrm{L}$ satisfy properties $\mathrm{L}_{1}, \mathrm{~L}_{2}$ on a nonempty set $\mathrm{E}, \mathrm{\omega}$ is weight function and $\mathrm{A}, \mathrm{B}$ are isotonic linear normalised functionals on $\mathrm{L}$, then for all $\mathrm{h} \cdot \omega \in \mathrm{L}$ such that $\Psi(h), \Psi(\eta+\zeta-A(h \cdot \omega)) \in L$ (so that $\eta \leqslant h(l) \leqslant \zeta$ for all $l \in E$ ), we have

$$
B(\Psi(\eta+\zeta-A(h \cdot \omega))) \leqslant \Psi(\eta)+\Psi(\zeta)-A(\Psi(h)) .
$$

Proof. Analogous to the proof of Theorem 2.2 we can prove it by using right hand side of the inequality (1.2) and using Theorem 1.1 instead of Theorem 2.1.

\section{Exponential convexity}

A real-valued function $k: I \rightarrow \mathbb{R}$ is m-exponentially convex if it is $m$-exponentially $\mathcal{J}$-convex and continuous on I. Hence it is an exponentially convex function (for detail see [19]).

Remark 3.1. A positive real-valued function $k: I \rightarrow \mathbb{R}$ is log-J-convex if and only if it is 2-exponentially $J$-convex. Converse is true provided that $k$ is also continuous.

Remark 3.2. If the divided difference $\left[t_{1}, t_{2} ; k\right] \geqslant 0$ for every $t_{1}, t_{2} \in I$, then $k$ is increasing on its domain. 
Lemma 3.3 ([10]). If $\Psi: \mathrm{I} \rightarrow \mathbb{R}$ is log-convex, then for $\mathrm{l}<\mathrm{r}<\mathrm{s}(\mathrm{l}, \mathrm{r}, \mathrm{s} \in \mathrm{I})$,

$$
(\Psi(r))^{s-l} \leqslant(\Psi(l))^{s-r}(\Psi(s))^{r-l} .
$$

Remark 3.4. We consider the following functionals under the assumption of Theorems 2.2 and 2.3, respectively.

$$
\begin{aligned}
\Omega(\Psi) & =\Psi(\eta)+\Psi(\zeta)-B(\Psi(A(h \cdot \omega)))-\Psi(\eta+\zeta-A(h)) \\
\Omega^{\prime}(\Psi) & =\Psi(\eta)+\Psi(\zeta)-A(\Psi(h))-B(\Psi(\eta+\zeta-A(h \cdot \omega))) .
\end{aligned}
$$

Then $\Omega(\Psi)$ and $\Omega^{\prime}(\Psi)$ are positive.

We construct m-exponentially convex functions and exponentially convex functions by applying an elegant method from [11]. The following theorem produces new m-exponentially convex functions.

Theorem 3.5. Let $\mathrm{J} \subset \mathbb{R}$ be an open interval, and $\Theta=\left\{\mathrm{g}_{\mathrm{l}} \mid \mathrm{l} \in \mathrm{J}\right\}$ is a family of functions defined on $\mathrm{I} \subset \mathbb{R}$, such that the function $\mathrm{l} \mapsto\left[\mathrm{t}_{1}, \mathrm{t}_{2}, \mathrm{t}_{3} ; \mathrm{g}_{\mathrm{l}}\right]$ is m-exponentially $\mathcal{J}$-convex on $\mathrm{J}$ for all three different points $\mathrm{t}_{1}, \mathrm{t}_{2}, \mathrm{t}_{3} \in \mathrm{I}$. Consider $\Omega(\Psi)$ as given in Remark 3.4. Then $\mathrm{l} \mapsto \Omega\left(\mathrm{g}_{\mathrm{l}}\right)$ is m-exponentially J-convex on $\mathrm{J}$. If the function $\mathrm{l} \mapsto \Omega\left(\mathrm{g}_{\mathrm{l}}\right)$ is continuous, then it is m-exponentially convex on $\mathrm{J}$.

Proof. Let $l_{i}, l_{j} \in J, l_{i j}=\frac{l_{i}+l_{j}}{2}$ and $a_{i}, a_{j} \in \mathbb{R}$ for $i, j \in\{1,2, \cdots, m\}(m \in \mathbb{N})$ and define the function $\Delta$ on I by

$$
\Delta(t)=\sum_{i, j=1}^{m} a_{i} a_{j} g_{l_{i j}}(t) .
$$

Then $\Delta$ being the linear combination of continuous functions, is a continuous function. By assumption the function $l \mapsto\left[t_{1}, t_{2}, t_{3} ; g_{l}\right]$ is m-exponentially $\mathcal{J}$-convex, therefore we have

$$
\left[t_{1}, t_{2}, t_{3} ; \Delta\right]=\sum_{i, j=1}^{m} a_{i} a_{j}\left[t_{1}, t_{2}, t_{3} ; g_{t_{i j}}\right] \geqslant 0,
$$

which implies that $\Delta$ is a convex function on I. Therefore we have $\Omega(\Delta) \geqslant 0$, which yields by the linearity of $\Omega$, that

$$
\sum_{i, j=1}^{m} a_{i} a_{j} \Omega\left(g_{i_{i j}}\right) \geqslant 0 .
$$

We conclude that the function $l \mapsto \Omega\left(g_{l}\right)$ is m-exponentially $\mathcal{J}$-convex function on $J$.

The following corollaries are consequence of above theorem.

Corollary 3.6. Let $\mathrm{J} \subset \mathbb{R}$ be an open interval, and $\Theta=\left\{\mathrm{g}_{\mathrm{l}} \mid \mathrm{l} \in \mathrm{J}\right\}$ is a family of functions defined on $\mathrm{I} \subset \mathbb{R}$,

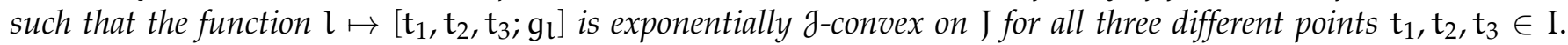
Consider $\Omega(\Psi)$ as given in Remark 3.4. Then $\mathrm{l} \mapsto \Omega\left(\mathrm{g}_{\mathrm{l}}\right)$ is an exponentially $\mathcal{J}$-convex function on $\mathrm{J}$. If the function $\mathrm{l} \mapsto \Omega\left(\mathrm{g}_{\mathrm{l}}\right)$ is continuous, then it is exponentially convex on $\mathrm{J}$.

Corollary 3.7. Let $\mathrm{J} \subset \mathbb{R}$ be an open interval, and $\Theta=\left\{g_{\mathrm{l}} \mid \mathrm{l} \in \mathrm{J}\right\}$ is a family of functions defined on $\mathrm{I} \subset \mathbb{R}$,

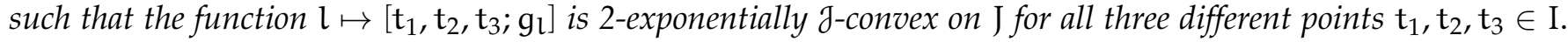
Consider $\Omega(\Psi)$ as given in Remark 3.4. Then $\mathrm{l} \mapsto \Omega\left(\mathrm{g}_{\mathrm{l}}\right)$ is 2-exponentially J-convex function on $\mathrm{J}$. If the function $\mathrm{l} \mapsto \Omega\left(\mathrm{g}_{\mathrm{l}}\right)$ is continuous, then it is 2-exponentially convex on $\mathrm{J}$, and thus log-convex, that is,

$$
\Omega^{l-r}\left(g_{s}\right) \leqslant \Omega^{l-s}\left(g_{r}\right) \Omega^{s-r}\left(g_{l}\right)
$$

for $\mathrm{l}, \mathrm{r}, \mathrm{s} \in \mathrm{J}$ such that $\mathrm{r}<\mathrm{s}<\mathrm{l}$. 
Proof. This is an immediate consequence of Theorem 3.5 and Remark 3.1.

Now we present different families of functions to investigate exponential convexity. The following lemma will be useful to construct new exponentially convex functions. Since the below mentioned result is the simple consequence of some basic examples and remarks given in [11], so we omit the proof.

\section{Lemma 3.8.}

(i) For $l>0$, let $\mathrm{f}_{\mathrm{l}}: \mathrm{I}=\mathbb{R} \rightarrow \mathbb{R}$ be defined by

$$
f_{l}(t)=\frac{1}{l^{2}} \exp (l t)
$$

Then $\mathrm{l} \mapsto \frac{\mathrm{d}^{2}}{\mathrm{dt}^{2}} \mathrm{f}_{\mathrm{l}}(\mathrm{t})$ is exponentially convex on $(0, \infty)$ for each $\mathrm{t} \in \mathrm{I}$.

(ii) For $l>1$, let $\mathrm{g}_{\mathrm{l}}: \mathrm{I}=\mathbb{R}^{+} \rightarrow \mathbb{R}$ be defined by

$$
g_{l}(t)=\frac{t^{l}}{l(l-1)}
$$

Then $\mathrm{l} \mapsto \frac{\mathrm{d}^{2}}{\mathrm{dt}^{2}} \mathrm{~g}_{\mathrm{l}}(\mathrm{t})$ is exponentially convex on $(1, \infty)$ for each $\mathrm{t} \in \mathrm{I}$.

(iii) For $l>1$, let $h_{l}: I=\mathbb{R}^{+} \rightarrow \mathbb{R}^{+}$be defined by

$$
h_{l}(t)=\frac{l^{-t}}{(\log l)^{2}} .
$$

Then $\mathrm{l} \mapsto \frac{\mathrm{d}^{2}}{\mathrm{dt}^{2}} \mathrm{~h}_{\mathrm{l}}(\mathrm{t})$ is exponentially convex on $(1, \infty)$ for each $\mathrm{t} \in \mathrm{I}$.

(iv) For $l>0$, let $k_{l}: I=\mathbb{R}^{+} \rightarrow \mathbb{R}^{+}$be defined by

$$
k_{l}(t)=\frac{1}{l} \exp (-t \sqrt{l}) .
$$

Then $\mathrm{l} \mapsto \frac{\mathrm{d}^{2}}{\mathrm{dt}^{2}} \mathrm{k}_{\mathrm{l}}(\mathrm{t})$ is exponentially convex on $(0, \infty)$ for each $\mathrm{t} \in \mathrm{I}$.

To define the basic inequality of log-convex functions we present positive functionals.

Remark 3.9. The following positive functionals are useful in defining the basic inequality of log-convex functions.

$$
\begin{aligned}
& \Lambda\left(f_{l}\right)=\frac{1}{l^{2}}(\exp (l \eta)+\exp (l \zeta)-B(\exp (l A(h \cdot \omega)))-\exp (l \eta+l \zeta-l A(h))), \\
& \Lambda\left(g_{l}\right)=\frac{1}{l(l-1)}\left(\eta^{l}+\zeta^{l}-B\left((A(h \cdot \omega))^{l}\right)-(\eta+\zeta-A(h))^{l}\right), \\
& \Lambda\left(h_{l}\right)=\frac{1}{(\log l)^{2}}\left(l^{-\eta}+l^{-\zeta}-B\left(l^{-A(h \cdot \omega)}\right)-l^{A(h)-\eta-\zeta}\right), \\
& \Lambda\left(k_{l}\right)=\frac{1}{l}(\exp (-\eta \sqrt{l})+\exp (-\zeta \sqrt{l})-B(\exp (-A(h \cdot \omega) \sqrt{l}))-\exp ((A(h)-\eta-\zeta) \sqrt{l})) .
\end{aligned}
$$

Theorem 3.10. Let $\Omega(\Psi)$ be the linear functional defined by (3.1) and define $\phi_{i}:(0, \infty) \rightarrow \mathbb{R}$ for $i=1,4$ and $\phi_{i}:(1, \infty) \rightarrow \mathbb{R}$ for $i=2,3$ by

$$
\phi_{1}(l)=\Lambda\left(f_{l}\right), \quad \phi_{2}(l)=\Lambda\left(g_{l}\right), \quad \phi_{3}(l)=\Lambda\left(h_{l}\right), \quad \phi_{4}(l)=\Lambda\left(k_{l}\right),
$$

where $\mathrm{f}_{l}, \mathrm{~g}_{\mathrm{l}}, \mathrm{h}_{\mathrm{l}}$ and $\mathrm{k}_{\mathrm{l}}$ are defined in Lemma 3.8. Then 
(i) The function $\phi_{i}$ are continuous on $(0, \infty)$ for $i=1,4$ and continuous on $(1, \infty)$ for $i=2,3$.

(ii) If $m \in \mathbb{N}, l_{1}, \cdots, l_{n} \in(0, \infty)$ for $i=1,4$ and $l_{1}, \cdots, l_{n} \in(1, \infty)$ for $i=2,3$, then the matrices

$$
\left[\phi_{i}\left(\frac{l_{j}+l_{k}}{2}\right)\right]_{j, k=1}^{m},
$$

are positive semidefinite.

(iii) The functions $\phi_{i}$ are exponentially convex on $(0, \infty)$ for $i=1,4$ and exponentially convex on $(1, \infty)$ for $i=2,3$.

(iv) If $l, r, s \in(0, \infty)$ for $i=1,4$ and $l, r, s \in(1, \infty)$ for $i=2,3$ are such that $l<r<s$, then

$$
\left(\phi_{i}(r)\right)^{s-l} \leqslant\left(\phi_{i}(l)\right)^{s-r}\left(\phi_{i}(s)\right)^{r-l},
$$

where $\phi_{i}(l)$ for $i=1,2,3,4$ are defined in Remark 3.9 .

Proof.

(i) The continuity of the functions $l \mapsto \phi_{i}(l)$ for $i \in\{1,2,3,4\}$ is obvious.

(ii) Let $m \in \mathbb{N}$ and $d_{j}, l_{j} \in \mathbb{R}(1 \leqslant j \leqslant m)$. Define the auxiliary function $\Delta_{1}$ on $I=\mathbb{R}$ by

$$
\Delta_{1}(t)=\sum_{j, k=1}^{m} d_{j} d_{k} f_{\frac{l_{j}+l_{k}}{2}}(t) .
$$

Since

$$
\Delta_{1}^{\prime \prime}(t)=\sum_{j, k=1}^{m} d_{j} d_{k} \frac{d^{2}}{d t^{2} \frac{f^{2}+l_{k}}{2}}(t) \geqslant 0
$$

for $\mathrm{t} \in \mathrm{I}$ by Lemma 3.8. This implies $\Delta_{1}$ is convex. Now Theorem 2.2 implies that $\Omega\left(\Delta_{1}\right) \geqslant 0$. This means that

$$
\left[\phi_{1}\left(\frac{l_{j}+l_{k}}{2}\right)\right]_{j, k=1}^{m}
$$

is a positive semidefinite matrix.

To prove the remaining positive semidefinite matrices, we can define the auxiliary functions $\Delta_{i}$ for $i=2,3,4$ in the similar manner.

(iii) and (iv) are simple consequences of (i), (ii) and Lemma 3.3.

Remark 3.11. We can construct similar results for the positive functional $\Omega^{\prime}(\Psi)$ defined in (3.2).

\section{Mean value theorems}

Now, we state the mean value theorems of Lagrange and Cauchy type.

The following lemma will be very useful.

Lemma 4.1 ([20]). Let $\Psi: I \rightarrow \mathbb{R}, I \subseteq \mathbb{R}$, be such that $\Psi \in \mathrm{C}^{2}(\mathrm{I}), \Psi^{\prime \prime}$ is bounded and $\mathrm{m}=\inf _{\mathrm{l} \in \mathrm{I}} \Psi^{\prime \prime}(\mathrm{l}), M=$ $\sup _{l \in \mathrm{I}} \Psi^{\prime \prime}(l)$. Then the functions $\Psi_{1}, \Psi_{2}: \mathrm{I} \rightarrow \mathbb{R}$ defined by

$$
\begin{aligned}
& \Psi_{1}(l)=\frac{M}{2} l^{2}-\Psi(l), \\
& \Psi_{2}(l)=\Psi(l)-\frac{m}{2} l^{2},
\end{aligned}
$$

are convex. 
Theorem 4.2. Let $\mathrm{L}$ satisfy properties $\mathrm{L}_{1}$ and $\mathrm{L}_{2}$ on a nonempty set $\mathrm{E}$, and let $\Psi: \mathrm{I} \rightarrow \mathbb{R}, \Psi \in \mathrm{C}^{2}(\mathrm{I})$, where $\mathrm{I}=[\eta, \zeta] \subseteq \mathbb{R}(-\infty<\eta<\zeta<\infty)$. If $\mathrm{A}, \mathrm{B}$ are isotonic linear normalised functionals and $\omega$ is a weight function (defined in (1.1)), then for all $h, h \cdot \omega \in L$ such that $\Psi(A(h \cdot \omega)),(A(h \cdot \omega))^{2} \in L$ there exists some $\gamma \in I$ such that the following holds

$$
\Psi(\eta)+\Psi(\zeta)-B(\Psi(A(h \cdot \omega)))-\Psi(\eta+\zeta-A(h))=\alpha \Psi^{\prime \prime}(\gamma),
$$

where

$$
\alpha=\frac{1}{2}\left[\eta^{2}+\zeta^{2}-(\eta+\zeta-A(h))^{2}-B\left([A(h \cdot \omega)]^{2}\right)\right] .
$$

Proof. Denote $M=\max _{l \in I} \Psi^{\prime \prime}(l)$ and $m=\min _{l \in I} \Psi^{\prime \prime}(l)$. Then by Lemma 4.1, the functions $\Psi_{1}, \Psi_{2}: I \rightarrow \mathbb{R}$ are convex. Since they are also continuous. Applying Theorem 2.2, we get

$$
\Psi(\eta)+\Psi(\zeta)-B(\Psi(A(h \cdot \omega)))-\Psi(\eta+\zeta-A(h)) \leqslant \alpha M,
$$

and

$$
\Psi(\eta)+\Psi(\zeta)-B(\Psi(A(h \cdot \omega)))-\Psi(\eta+\zeta-A(h)) \geqslant \alpha m .
$$

Now combining these two inequalities and since $\Psi^{\prime \prime}$ is continuous, there exists $\gamma \in \mathrm{I}\left(\mathrm{m} \leqslant \Psi^{\prime \prime}(\gamma) \leqslant M\right)$ such that (4.1) holds.

Theorem 4.3. Let $\mathrm{L}$ satisfy properties $\mathrm{L}_{1}$ and $\mathrm{L}_{2}$ on a nonempty set $\mathrm{E}$, and let $\Phi, \Psi: \mathrm{I} \rightarrow \mathbb{R}, \Phi, \Psi \in \mathrm{C}^{2}(\mathrm{I})$, where $\mathrm{I}=[\eta, \zeta] \subseteq \mathbb{R}(-\infty<\eta<\zeta<\infty)$. If $\mathrm{A}, \mathrm{B}$ are isotonic linear normalised functionals and $\omega$ is a weight function (defined in (1.1)), then for all $h, h \cdot \omega \in L$ such that $\Phi(A(h \cdot \omega)), \Psi(A(h \cdot \omega)),(A(h \cdot \omega))^{2} \in L$ and $\eta^{2}+\zeta^{2}-(\eta+\zeta-A(h))^{2}-B\left([A(h \cdot \omega)]^{2}\right) \neq 0$ there exists some $\gamma \in I$ such that the following holds

$$
\begin{aligned}
& \Psi^{\prime \prime}(\gamma)[\Phi(\eta)+\Phi(\zeta)-B(\Phi(A(h \cdot \omega)))-\Phi(\eta+\zeta-A(h))] \\
& =\Phi^{\prime \prime}(\gamma)[\Psi(\eta)+\Psi(\zeta)-B(\Psi(A(h \cdot \omega)))-\Psi(\eta+\zeta-A(h))] .
\end{aligned}
$$

Proof. Consider the function $k \in C^{2}(I)$ defined as $k=c_{1} \Phi-c_{2} \Psi$, where $c_{1}$ and $c_{2}$ are defined by

$$
c_{1}=\Psi(\eta)+\Psi(\zeta)-B(\Psi(A(h \cdot \omega)))-\Psi(\eta+\zeta-A(h)),
$$

and

$$
c_{2}=\Phi(\eta)+\Phi(\zeta)-B(\Phi(A(h \cdot \omega)))-\Phi(\eta+\zeta-A(h)) .
$$

Since $k \in C^{2}(I)$, now by applying Theorem 4.2 on the function $k$, it follows that there exists some $\gamma \in I$ such that the following holds

$$
k(\eta)+k(\zeta)-B(k(A(h \cdot \omega)))-k(\eta+\zeta-A(h))=\alpha k^{\prime \prime}(\gamma) .
$$

The left-hand side of this equation equals to zero, since $\alpha \neq 0$, so we have that $k^{\prime \prime}(\gamma)=0$. Thus the assertion of our theorem follows directly.

Similarly we can define mean value theorems for Theorem 2.3. Here we omit the proofs.

Theorem 4.4. Let $\mathrm{L}$ satisfy properties $\mathrm{L}_{1}$ and $\mathrm{L}_{2}$ on a nonempty set $\mathrm{E}$, and let $\Psi: \mathrm{I} \rightarrow \mathbb{R}, \Psi \in \mathrm{C}^{2}(\mathrm{I})$, where $\mathrm{I}=[\eta, \zeta] \subseteq \mathbb{R}(-\infty<\eta<\zeta<\infty)$. If $\mathrm{A}, \mathrm{B}$ are isotonic linear normalised functionals and $\omega$ is a weight function (defined in (1.1)), then for all $h \cdot \omega \in \mathrm{L}$ such that $\Psi(\eta+\zeta-A(h \cdot \omega)), \Psi(h),(\eta+\zeta-A(h \cdot \omega))^{2}, h^{2} \in L$ there exists some $\gamma \in$ I such that the following holds

$$
\Psi(\eta)+\Psi(\zeta)-A(\Psi(h))-B(\Psi(\eta+\zeta-A(h \cdot \omega)))=\beta \Psi^{\prime \prime}(\gamma),
$$

where

$$
\beta=\frac{1}{2}\left[\eta^{2}+\zeta^{2}-A\left(h^{2}\right)-B\left([\eta+\zeta-A(h \cdot \omega)]^{2}\right)\right] .
$$


Theorem 4.5. Let $\mathrm{L}$ satisfy properties $\mathrm{L}_{1}$ and $\mathrm{L}_{2}$ on a nonempty set $\mathrm{E}$, and let $\Phi, \Psi: \mathrm{I} \rightarrow \mathbb{R}, \Phi, \Psi \in \mathrm{C}^{2}(\mathrm{I})$, where $\mathrm{I}=[\eta, \zeta] \subseteq \mathbb{R}(-\infty<\eta<\zeta<\infty)$. If $\mathrm{A}, \mathrm{B}$ are isotonic linear normalised functionals and $\omega$ is a weight function (defined in (1.1)), then for all $h \cdot \omega \in L$ such that $\Phi(\eta+\zeta-A(h \cdot \omega)), \Psi(\eta+\zeta-A(h \cdot \omega)), \Phi(h), \Psi(h),(\eta+$ $\zeta-A(h \cdot \omega))^{2}, h^{2} \in L$ and $\eta^{2}+\zeta^{2}-A\left(h^{2}\right)-B\left([\eta+\zeta-A(h \cdot \omega)]^{2}\right) \neq 0$ there exists some $\gamma \in$ I such that the following holds

$$
\begin{aligned}
& \Psi^{\prime \prime}(\gamma)[\Phi(\eta)+\Phi(\zeta)-A(\Phi(h))-B(\Phi(\eta+\zeta-A(h \cdot \omega)))] \\
& =\Phi^{\prime \prime}(\gamma)[\Psi(\eta)+\Psi(\zeta)-A(\Psi(h))-B(\Psi(\eta+\zeta-A(h \cdot \omega)))]
\end{aligned}
$$

\section{Acknowledgment}

This research is supported by National University of Sciences and Technology (NUST), Islamabad and Higher Education Commission of Pakistan.

\section{References}

[1] M. Adil Khan, T. Ali, A. Kılıçman, Q. Din, Refinements of Jensen's inequality for convex functions on the co-ordinates in a rectangle from the plane, Filomat, 30 (2016), 803-814. 1

[2] M. Adil Khan, G. Ali Khan, T. Ali, A. Kılıçman, On the refinement of Jensen's inequality, Appl. Math. Comput., 262 (2015), 128-135.

[3] M. Adil Khan, J. Khan, J. Pečarić, Generalization of Jensen's and Jensen-Steffensen's inequalities by generalized majorization theorem, J. Math. Inequal., (2017), to appear. 1

[4] M. Anwar, N. Latif, J. Pečarić, Positive semidefinite matrices, exponential convexity for majorization, and related Cauchy means, J. Inequal. Appl., 2010 (2010), 19 pages. 1

[5] P. R. Beesack, J. Pečarić, On Jessen's inequality for convex functions, J. Math. Anal. Appl., 110 (1985), 536-552. 1

[6] S. N. Bernstein, Sur les fonctions absolument monotones, (French) Acta Math., 52 (1929), 1-66. 1

[7] S. I. Butt, R. Jakšić, L. Kvesić, J. Pečarić, n-exponential convexity of weighted Hermite-Hadamard's inequality, J. Math. Inequal., 8 (2014), 299-311. 1

[8] S. S. Dragomir, M. Adil Khan, A. Abathun, Refinement of the Jensen integral inequality, Open Math., 14 (2016), 221-228. 1

[9] S. Iqbal, K. Krulić Himmelreich, J. Pečarić, D. Pokaz, n-exponential convexity of Hardy-type and Boas-type functionals, J. Math. Inequal., 7 (2103), 739-750. 1

[10] J. Jakšetić, R. Naeem, J. Pečarić, Exponential convexity for Jensen's inequality for norms, J. Inequal. Appl., 2016 (2016), 8 pages. $1,3.3$

[11] J. Jakšetić, J. Pečarić, Exponential convexity method, J. Convex Anal., 20 (2013), 181-197. 1, 3, 3

[12] B. Jessen, Bemærkninger om konvekse Funktioner og Uligheder imellem Middelværdier, I, Mat. Tidsskrift B, (1931), 17-28. 1

[13] S. Khalid, J. Pečarić, On the refinements of the integral Jensen-Steffensen inequality, J. Inequal. Appl., 2013 (2013), 18 pages. 1

[14] K. A. Khan, A. Nosheen, J. Pečarić, n-exponential convexity of some dynamic Hardy-type functionals, J. Math. Inequal., 8 (2014), 331-347.

[15] A. R. Khan, J. Pečarić, M. R. Lipanović, n-exponential convexity for Jensen-type inequalities, J. Math. Inequal., 7 (2013), 313-335. 1

[16] A. M. Mercer, An "error term" for the Ky Fan inequality, J. Math. Anal. Appl., 220 (1998), 774-777. 1

[17] A. M. Mercer, Some new inequalities involving elementary mean values, J. Math. Anal. Appl., 229 (1999), 677-681. 1

[18] R. Naeem, m-Exponential convexity of refinements of Hermite-Hadamards inequality, Proc. Pakistan Acad. Sci., 54 (2017), 197-205. 1

[19] J. Pečarić, J. Perić, Improvements of the Giaccardi and the Petrovi inequality and related Stolarsky type means, An. Univ. Craiova Ser. Mat. Inform., 39 (2012), 65-75. 1, 3

[20] J. E. Pečarić, F. Proschan, Y. L. Tong, Convex functions, partial orderings, and statistical applications, Mathematics in Science and Engineering, Academic Press, Inc., Boston, MA, (1992). 1, 4.1

[21] D. V. Widder, Necessary and sufficient conditions for the representation of a function by a doubly infinite Laplace integral, Bull. Amer. Math. Soc., 40 (1934), 321-326. 1 